\title{
Triptolide-Mediated Apoptotic Cell Death Accelerated by Metformin Co-Treatment in MiaPaca-2 Cells ${ }^{\dagger}$
}

\author{
Zeynep Elif Apaydin, Ulku Gungor, Burcu Ayhan-Sahin, Pınar Obakan-Yerlikaya, \\ Elif-Damla Arisan and Ajda Coker-Gurkan * \\ Department of Molecular Biology and Genetics, Istanbul Kultur University, 34156 Istanbul, Turkey; \\ zeynepapaydin93@gmail.com (Z.E.A.); ulku.gungor1460@gmail.com (U.G.); burcu.sahin@iku.edu.tr (B.A.-S.); \\ p.obakan@iku.edu.tr (P.O.-Y.); d.arisan@iku.edu.tr (E.-D.A.) \\ * Correspondence: a.coker@iku.edu.tr; Tel.: +90-212-498-4565 \\ + Presented at the 3rd International conference on Natural Products for Cancer Prevention and Therapy, \\ Kayseri, Turkey, 18-20 December 2019.
}

Published: 25 December 2019

\begin{abstract}
Pancreatic cancer is the fourth most common cause of cancer death worldwide with limited therapeutic potential and low survival rate. A natural agent; triptolide (diterpenoid triepoxide), induce cell viability loss, growth inhibition and apoptotic cell death in various cancer cells such as breast, prostate and pancreatic cancer. Metformin, a common therapeutic agent for type II Diabetes mellitus, has been shown to induce apoptotic cell death in pancreatic cancer via modulating various pathways such as PI3K/Akt pathways. In this study, our aim was to investigate the potential additional effect of metformin on triptolide-induced apoptotic cell death in MiaPaCa2 cells. Drugs-mediated cell viability loss, growth inhibition and colony formation potential were examined by MTT, trypan blue exclusion, colony formation, hanging drop assays. Drugs triggered apoptotic cell death was determined by PI FACS flow analysis. According to MTT cell viability assay, $20 \mathrm{nM}$ triptolide decreased cell viability by $78 \%$, but triptolide and metformin co-treatment decreased cell viability by $57 \%$. Metformin co-treatment increased the triptolide-mediated cell growth inhibition, and prevention of colony formation in MiaPaCa-2 cells. In addition, co-treatment increased triptolide-triggered mitochondrial membrane potential loss, cell death and ROS generation. In conclusion, metformin co-treatment accelerated triptolide-triggered apoptotic cell death in MiaPaCa-2 cells.
\end{abstract}

Keywords: pancreatic cancer; triptolide; metformin; apoptosis

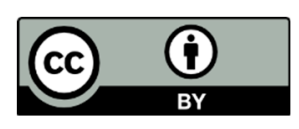

(C) 2019 by the authors. Licensee MDPI, Basel, Switzerland. This article is an open access article distributed under the terms and conditions of the Creative Commons Attribution (CC BY) license (http://creativecommons.org/licenses/by/4.0/). 